\title{
The Free Radical Theory of Ageing: Where are We 60 Years Later?
}

\author{
Nagba Yendoubé Gbandjaba ${ }^{1 *}$, Pakoupati Boyode ${ }^{1}$, Kafui Kpegba $^{1}$ and Abdelouahed Khalil ${ }^{2}$ \\ ${ }^{1}$ Laboratoire de Chimie Organique et des Substances Naturelles, Université de Lomé, Lomé, Togo \\ ${ }^{2}$ Centre de Recherche sur le Vieillissement, Département de Médecine, Université de Sherbrooke,Sherbrooke, Québec, Canada
}

*Corresponding author: Gbandjaba Nagba Yendoubé, Laboratoire de Chimie Organique et des Substances Naturelles, Université de Lomé, Lomé, Togo

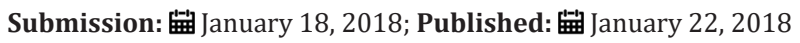

\section{Editorial}

Our average life expectancy is getting longer at a rate of 3 months every year. By day, we win 6 hours of life expectancy. Human global average life expectancy is 71 years write now. One century before, it was 30 years. In other words, the advancing knowledge of better hygiene, biomedicine, and elimination of the most infectious diseases occurring in youth, vaccine and the good nutrition has led us to discover the aging process and the lengthening of life expectancy. The immediate consequence of the extended life expectancy is represented by the increasing number of older people in developed countries.

Ageing is not a disease but is commonly defined as the accumulation of diverse deleterious changes occurring in cells and tissues with advancing age that are responsible for the increased risk of diseases and death [1]. Ageing is becoming the major risk factor for diseases and death for humans after the age of 28 years in the developed country.

The Free Radical Theory of Ageing (FRTA) has got 60 years in 2016. But over the last decade, a small controversy appeared to fail the FRTA [2-4]. The authors of the controversy said that, in some cases, generation of Reactive Oxygen Species (ROS) increases longevity [5]. By addition, antioxidants supplementation did not decrease the incidence of many age associated diseases but raises the risk of death. The detractors of the FRTA are in favor of the unified theory of ageing because it is a complex process involving defects in various cellular components [6].

Denham Harman is the author of the FRTA [3]. Harman, pioneer of the research on ageing postulated that "aging and the degenerative diseases associated with it are attributed basically to the deleterious side attacks of free radicals on cell constituents and on the connected tissues". About three hundred fifty theories of ageing have been proposed and are classified in three groups:
A. The genetic mutation theories
B. The wear and tear theories

\section{And the cellular waste accumulation theories.}

It is important to know that, some theories can be included in two groups. This is applied to the FRTA which shares the cellular waste accumulation theories and the genetic mutation theories. The free radical theory of ageing is one of the most prominent and well studied among the 350 theories [4].

Mitochondrion is the key organelle involved in ageing because it is the source of free radicals production [2]. The Mitochondrial Free Radical Theory of Aging (MFRTA) proposes that mitochondrial free radicals, produced during normal metabolism, cause oxidative damage. According to MFRTA, the accumulation of this oxidative damage is the main driving force in the aging process [7].

Oxygen is vital for our life. Without it we will die. Around $90 \%$ of oxygen we breathe is used to produce energy. But approximatively 5 to $10 \%$ of the oxygen is very susceptible to free radical formation [8], which constitutes the dark side of oxygen. The destructive nature of oxygen is oxygen paradox. Free radicals are molecules with impaired electrons. Free radicals are also unstable molecules that damage cells. Oxygen free radicals or oxyradicals include peroxide anion radical, singlet oxygen, and hydroxyl radical. Oxyradicals has been implicated in many disease processes. Free radicals are also derived from Reactive Nitrogen Species (RNS).

Antioxidants inhibit free radicals bad effects. For example, Vitamin E, which is defined as the chain-breaking antioxidant, disarms free radicals and is transformed to oxidized form. Vitamin C recycles oxidized Vitamin E and is transformed to Vitamin C radicals. Glutathione neutralizes Vitamin $C$ radicals. That is why, it is important to match all kind of antioxidant in our food [9].

In many species like rats or human, we observed that, females live longer than males [10]. The women live 5 years longer than man in human. We could attribute the increasing life expectancy in women to the fact that, they lose the Iron every month during their menstrual cycle $[11,12]$. Iron is highly involved in free radical 
production. Women during their lifetime has seen 2250 days of rules, around an estimated 450 periods, started menstruating at the puberty and going through the menopause. This may explain why women produce fewer free radicals by comparison to man [11]. This point of view is in favor of the free radical theory of ageing $[3,7]$.

\section{References}

1. Hekimi S, Lapointe J, Wen Y (2011) Taking a "good" look at free radicals in the aging process. Trends Cell Biol 21(10): 569-576.

2. Lambert AJ, Brand MD (2007) Research on mitochondria and aging, 2006-2007. Aging Cell 6(4): 417-420.

3. Harman D (1956) Aging: a theory based on free radical and radiation chemistry. J Gerontol 11(3): 298-300.

4. Vina J, Borras C, Miquel J (2007) Theories of ageing. IUBMB Life 59(4-5): 249-254.

5. Miwa S, Riyahi K, Partridge L, Brand MD (2004) Lack of correlation between mitochondrial reactive oxygen species production and life span in Drosophila. Ann N Y Acad Sci 1019: 388-391.
6. Kelly DP (2011) Cell biology: ageing theories unified. Nature 470(7334): 342-343.

7. Miquel J, Economos AC, Fleming J, Johnson JE Jr (1980) Mitochondrial role in cell aging. Exp Gerontol 15(6): 575-591.

8. Cadenas E, Davies KJ (2000) Mitochondrial free radical generation, oxidative stress, and aging. Free Radic Biol Med 29(3-4): 222-230.

9. Pauling L (1974) Are recommended daily allowances for vitamin C adequate? Proc Natl Acad Sci U S A 71(11): 4442-4446.

10. Vina J, Borras C, Gambini J, Sastre J, Pallardo FV (2005) Why females live longer than males? Importance of the upregulation of longevityassociated genes by oestrogenic compounds. FEBS Lett 579(12): 25412545.

11. Ristow M, Zarse K (2010) How increased oxidative stress promotes longevity and metabolic health: the concept of mitochondrial hormesis (mitohormesis). Exp Gerontol 45(6): 410-418.

12. Mascitelli L, Pezzetta F, Goldstein MR (2010) Body iron stores and gender differences in risk factors for coronary heart disease. Maturitas 66(1): 107 